\title{
On a characterization of the essential spectra of some matrix operators and application to two-group transport operators
}

\author{
Salma Charfi · Aref Jeribi
}

Received: 30 January 2008 / Published online: 6 August 2008

(C) The Author(s) 2008. This article is published with open access at Springerlink.com

\begin{abstract}
In this paper, we investigate the essential approximate point spectrum and the essential defect spectrum of a $2 \times 2$ block operator matrix on a Banach space. Furthermore, we apply the obtained results to two-group transport operators in the Banach space $L_{p}([-a, a] \times$ $[-1,1]) \times L_{p}([-a, a] \times[-1,1]), a>0, p \geq 1$.
\end{abstract}

\section{Introduction}

In this article, we are concerned with the essential spectra of operators defined by a $2 \times 2$ block operator matrix,

$$
\mathcal{A}_{0}:=\left(\begin{array}{ll}
A & B \\
C & D
\end{array}\right) \text {. }
$$

In general, the operators occurring in the representation (1.1) are unbounded. $A$ acts on the Banach space $X$ and has the domain $\mathcal{D}(A), D$ is defined on $\mathcal{D}(D)$ and acts on the Banach space $Y$ and the intertwining operators $B$ and $C$ are defined on the domains $\mathcal{D}(B)$ and $\mathcal{D}(C)$, respectively, and act between these spaces. Then the operator $\mathcal{A}_{0}$ is defined on the domain $[\mathcal{D}(A) \cap \mathcal{D}(C)] \times[\mathcal{D}(D) \cap \mathcal{D}(B)]$. Note that, the operator $\mathcal{A}_{0}$ need to be closed, or the domain of this operator can be determined by an additional relation between the components $x$ and $y$ of its elements. In [2], it is shown under some conditions that $\mathcal{A}_{0}$ is closable and its closure will be denoted by the operator $\mathcal{A}$.

Let $X$ and $Y$ be two Banach spaces. We denote by $\mathcal{L}(X, Y)(\operatorname{resp} . \mathcal{C}(X, Y))$ the set of all bounded (resp. closed, densely defined) linear operators from $X$ into $Y$ and we denote by $\mathcal{K}(X, Y)$ the subspace of compact operators from $X$ into $Y$. For $T \in \mathcal{C}(X, Y)$, we write $\mathcal{D}(T) \subset X$ for the domain, $\mathcal{N}(T) \subset X$ for the null space and $\mathcal{R}(T) \subset Y$ for the range of $T$.

S. Charfi · A. Jeribi $(\bowtie)$

Département de Mathématiques, Université de Sfax, Faculté des Sciences de Sfax,

Route de Soukra Km 3.5, B.P. 1171, 3000 Sfax, Tunisia

e-mail: Aref.Jeribi@fss.rnu.tn 
The nullity, $\alpha(T)$, of $T$ is defined as the dimension of $\mathcal{N}(T)$ and the deficiency, $\beta(T)$, of $T$ is defined as the codimension of $\mathcal{R}(T)$ in $Y$.

Let $\sigma(T)$ (resp. $\rho(T)$ ) denote the spectrum (resp. the resolvent set) of $T$. The set of upper semi-Fredholm operators is defined by

$$
\Phi_{+}(X, Y):=\{T \in \mathcal{C}(X, Y) \text { such that } \alpha(T)<\infty \text { and } \mathcal{R}(T) \text { is closed in } \mathrm{Y}\}
$$

and the set of lower semi-Fredholm operators is defined by

$$
\Phi_{-}(X, Y):=\{T \in \mathcal{C}(X, Y) \text { such that } \beta(T)<\infty \text { and } \mathcal{R}(T) \text { is closed in } \mathrm{Y}\} .
$$

$\Phi(X, Y):=\Phi_{+}(X, Y) \cap \Phi_{-}(X, Y)$ denote the set of Fredholm operators from $X$ into $Y$ and $\Phi_{ \pm}(X, Y):=\Phi_{+}(X, Y) \cup \Phi_{-}(X, Y)$ the set of semi-Fredholm operators from $X$ into $Y$. While the number $i(T):=\alpha(T)-\beta(T)$ is called the index of $T$, for $T \in \Phi_{ \pm}(X, Y)$.

If $X=Y$ then $\mathcal{L}(X, Y), \mathcal{C}(X, Y), \mathcal{K}(X, Y), \Phi(X, Y), \Phi_{+}(X, Y), \Phi_{-}(X, Y)$ and $\Phi_{ \pm}(X, Y)$ are replaced by $\mathcal{L}(X), \mathcal{C}(X), \mathcal{K}(X), \Phi(X), \Phi_{+}(X), \Phi_{-}(X)$ and $\Phi_{ \pm}(X)$, respectively. A complex number $\lambda$ is in $\Phi_{+T}, \Phi_{-T}, \Phi_{ \pm T}$ or $\Phi_{T}$ if $\lambda-T$ is in $\Phi_{+}(X), \Phi_{-}(X), \Phi_{ \pm}(X)$ or $\Phi(X)$, respectively.

In this work, we are concerned with the following essential spectra:

$$
\begin{aligned}
& \sigma_{e g}(T):=\left\{\lambda \in \mathbb{C} \text { such that } \lambda-T \notin \Phi_{+}(X)\right\}:=\mathbb{C} \backslash \Phi_{+T}, \\
& \sigma_{\text {ew }}(T):=\left\{\lambda \in \mathbb{C} \text { such that } \lambda-T \notin \Phi_{-}(X)\right\}:=\mathbb{C} \backslash \Phi_{-T}, \\
& \sigma_{\text {ess }}(T):=\mathbb{C} \backslash \rho_{\text {ess }}(T) \text {, } \\
& \sigma_{b}(T):=\sigma(T) \backslash \sigma_{d}(T), \\
& \sigma_{\text {eap }}(T):=\mathbb{C} \backslash \rho_{\text {eap }}(T) \text {, } \\
& \sigma_{e \delta}(T):=\mathbb{C} \backslash \rho_{e \delta}(T),
\end{aligned}
$$

where $\rho_{\text {ess }}(T):=\left\{\lambda \in \Phi_{T}\right.$ such that $\left.i(\lambda-T)=0\right\}$ and $\sigma_{d}(T)$ is the set of isolated points $\lambda$ of the spectrum such that the corresponding Riesz projectors $P_{\lambda}$ are finite dimensional. The characterization of the sets $\rho_{e a p}($.$) and \rho_{e \delta}($.$) is given by Jeribi and Moalla [20] as follows$

$$
\rho_{\text {eap }}(T):=\left\{\lambda \in \mathbb{C} \text { such that } \lambda-T \in \Phi_{+}(X) \text { and } i(\lambda-T) \leq 0\right\},
$$

and

$$
\rho_{e \delta}(T):=\left\{\lambda \in \mathbb{C} \text { such that } \lambda-T \in \Phi_{-}(X) \text { and } i(\lambda-T) \geq 0\right\} .
$$

We call $\sigma_{e g}($.$) and \sigma_{e w}($.$) the Gustafson and Weidmann essential spectra [12] and \sigma_{e s s}($. the Schechter essential spectrum [12,14-16,26,27]. $\sigma_{\text {eap }}($.$) is the essential approximate$ point spectrum $[20,24,25]$ and $\sigma_{e \delta}($.$) is the essential defect spectrum [20,25,28] . \sigma_{b}($.$) is$ the Browder spectrum [18]. In the next, we will denote by $\rho_{b}():.=\mathbb{C} \backslash \sigma_{b}($.$) the Browder$ resolvent set.

In recent years, a number of papers have been devoted to study the essential spectra of block operator matrices acting in a product of Banach spaces. The situation where the domains of the diagonal operators satisfy $\mathcal{D}(A) \subset \mathcal{D}(C)$ and $\mathcal{D}(B) \subset \mathcal{D}(D)$ was considered by the authors in $[1,30]$ to study the Wolf essential spectrum [34]. They have assumed the compactness condition for the operators $(\lambda-A)^{-1}$ (see [1]) and $C(\lambda-A)^{-1}$ and $\left((\lambda-A)^{-1} B\right)^{*}$ (see [30]) for some (and hence for all) $\lambda$ in the resolvent set $\rho(A)$, whereas in the paper of [4], it is assumed that only $(\lambda-A)^{-1}, \lambda \in \rho(A)$, belongs to a nonzero two-sided closed ideal $\mathcal{I}(X) \subset \mathcal{F}(X)$ of $\mathcal{L}(X)$ where $\mathcal{F}(X)$ is the set of Fredholm perturbations (see Sect. 2). Thus, Moalla et al. [19] extend the obtained results into a large class of operators and describe many essential spectra of $\mathcal{A}$ and they apply their results to describe the essential 
spectra of two-group transport operators with general boundary conditions in $L_{p}$-spaces. But, to determine the essential spectra of $\mathcal{A}$, they must absolutely know the one of the entry $A$ of the matrix (1.1). In [2], A. Bátkai, P. Binding, A. Dijksma, R. Hryniv and H. Langer give a generalization of these results and describe the essential spectrum of $\mathcal{A}$. They have assumed that $\mathcal{D}(A) \subset \mathcal{D}(C)$ and that the intersection of the domains of the operators $B$ and $D$ is sufficiently large. Moreover, the domains of the operator matrix is defined by an additional relation of the form $\Gamma_{X}(x)=\Gamma_{Y}(y)$ between the two components of its elements. In fact, they have supposed that the operator $C\left(A_{1}-\lambda\right)^{-1}$ is compact for some (and hence for all) $\lambda$ in the resolvent set of $A_{1}$ where $A_{1}:=\left.A\right|_{\mathcal{D}(A) \cap \mathcal{N}\left(\Gamma_{X}\right)}$. However, in the classical transport theory, in $L_{1}$-spaces this operator is weakly compact (see Sect. 4 ). Therefore, their results cannot be applied in our work. From this problem, we have the idea to extend these results and we concern ourselves exclusively with the investigation of the essential approximate $\sigma_{\text {eap }}(\mathcal{A})$ and the essential defect spectrum $\sigma_{e \delta}(\mathcal{A})$. Indeed, the use of the Browder resolvent and the lower-upper factorization given by [18] allow us to formulate and give some supplements to many results presented in [2]. By comparison with the papers of [4,19], we mention that we can determine the essential spectra of matrix $\mathcal{A}$ without having the essential spectra of the operator $A$, but we know only the one of its restriction $A_{1}$ and we will give in our work an application in transport theory which is more general than the one provided in [19].

This paper is divided into four sections. In the next section, we give some preliminary results and notations used in the sequel of the paper. In Sect. 3 we introduce the assumptions $(H 1)-(H 8)$ to be imposed on the entries of the matrix (1.1) and we give a characterization of its essential approximate point spectrum and its essential defect spectrum. In the last section, we apply the obtained results to describe $\sigma_{e a p}($.$) and \sigma_{e \delta}($.$) of a class of transport equations$ acting in the Banach space $X_{p} \times X_{p}, 1 \leq p<\infty$, where

$$
X_{p}:=L_{p}([-a, a] \times[-1,1]), \quad a>0 .
$$

We will consider the following operator

$$
\mathcal{A}=\mathcal{T}+\mathcal{K},
$$

where

$$
\mathcal{T} \psi=\left(\begin{array}{cc}
-\xi \frac{\partial \psi_{1}}{\partial x}-\sigma_{1}(\xi) \psi_{1} & 0 \\
0 & -\xi \frac{\partial \psi_{2}}{\partial x}-\sigma_{2}(\xi) \psi_{2}
\end{array}\right)=\left(\begin{array}{cc}
T_{1} & 0 \\
0 & T_{2}
\end{array}\right)\left(\begin{array}{l}
\psi_{1} \\
\psi_{2}
\end{array}\right)
$$

and

$$
\mathcal{K}=\left(\begin{array}{cc}
0 & K_{12} \\
K_{21} & K_{22}
\end{array}\right)
$$

with $K_{12}, K_{21}$ and $K_{22}$ are bounded linear operators defined on $X_{p}$ by

$$
\left\{\begin{array}{l}
K_{i j}: X_{p} \longrightarrow X_{p} \\
\psi \longrightarrow \int_{-1}^{1} \kappa_{i j}\left(x, \xi, \xi^{\prime}\right) \psi\left(x, \xi^{\prime}\right) d \xi^{\prime}
\end{array}\right.
$$


and the kernels $\kappa_{12}(., .,),. \kappa_{21}(., .,$.$) and \kappa_{22}(., .,$.$) are assumed to be measurable. The$ operator $T_{1}$ is defined by

$$
\left\{\begin{aligned}
T_{1}: \mathcal{D}\left(T_{1}\right) \subseteq X_{p} \longrightarrow X_{p} \\
\psi \longrightarrow T_{1} \psi(x, \xi)=-\xi \frac{\partial \psi}{\partial x}(x, \xi)-\sigma_{1}(\xi) \psi(x, \xi) \\
\mathcal{D}\left(T_{1}\right)=\left\{\psi \in X_{p} \text { such that } \xi \frac{\partial \psi}{\partial x} \in X_{p}\right\}
\end{aligned}\right.
$$

and $T_{2}$ is the streaming operator defined by

$$
\left\{\begin{aligned}
T_{2}: \mathcal{D}\left(T_{2}\right) \subseteq X_{p} \longrightarrow X_{p} \\
\psi \longrightarrow T_{2} \psi(x, \xi)=-\xi \frac{\partial \psi}{\partial x}(x, \xi)-\sigma_{2}(\xi) \psi(x, \xi) \\
\mathcal{D}\left(T_{2}\right)=\left\{\psi \in X_{p} \quad \text { such that } \xi \frac{\partial \psi}{\partial x} \in X_{p} \quad \text { and } \psi^{i}=H\left(\psi^{0}\right)\right\},
\end{aligned}\right.
$$

where $\sigma(.) \in L^{\infty}(-1,1), \psi^{0}, \psi^{i}$ represent the outgoing and the incoming fluxes related by the boundary operator $H$. The function $\psi(x, \xi)$ represents the number density of gas particles having the position $x$ and the direction cosine of propagation $\xi$. The variable $\xi$ may be thought of as the cosine of the angle between the velocity of particles and the $x$-direction. The function $\sigma_{j}(),. j=1,2$, is a measurable function called the collision frequency.

\section{Notations and preliminaries results}

In this section, we recall some definitions and we give some lemmas that we will need in the sequel.

Definition 2.1 Let $X$ and $Y$ be two Banach spaces and let $F \in \mathcal{L}(X, Y)$.

(i) $F$ is called a Fredholm perturbation if $T+F \in \Phi(X, Y)$ whenever $T \in \Phi(X, Y)$.

(ii) $F$ is called an upper (resp. lower) semi-Fredholm perturbation if $T+F \in \Phi_{+}(X, Y)$ (resp. $\left.\Phi_{-}(X, Y)\right)$ whenever $T \in \Phi_{+}(X, Y)$ (resp. $\left.\Phi_{-}(X, Y)\right)$.

The sets of Fredholm, upper and lower semi-Fredholm perturbations are denoted by $\mathcal{F}(X, Y), \mathcal{F}_{+}(X, Y)$ and $\mathcal{F}_{-}(X, Y)$, respectively. If in Definition 2.1 we replace $\Phi(X, Y)$, $\Phi_{+}(X, Y)$ and $\Phi_{-}(X, Y)$ by $\Phi^{b}(X, Y):=\Phi(X, Y) \cap \mathcal{L}(X, Y), \Phi_{+}^{b}(X, Y):=\Phi_{+}(X, Y) \cap$ $\mathcal{L}(X, Y)$ and $\Phi_{-}^{b}(X, Y):=\Phi_{-}(X, Y) \cap \mathcal{L}(X, Y)$ we obtain the sets $\mathcal{F}^{b}(X, Y), \mathcal{F}_{+}^{b}(X, Y)$ and $\mathcal{F}_{-}^{b}(X, Y)$. These classes of operators are introduced and investigated by Gohberg et al. [10]. Recently, it is shown in [3] that $\mathcal{F}^{b}(X, Y), \mathcal{F}_{+}^{b}(X, Y)$ and $\mathcal{F}_{-}^{b}(X, Y)$ are closed subsets of $\mathcal{L}(X, Y)$ and if $X=Y$, then $\mathcal{F}^{b}(X):=\mathcal{F}^{b}(X, X), \mathcal{F}_{+}^{b}(X):=\mathcal{F}_{+}^{b}(X, X)$ and $\mathcal{F}_{-}^{b}(X):=\mathcal{F}_{-}^{b}(X, X)$ are closed two-sided ideals of $\mathcal{L}(X)$.

Proposition 2.1 [3, Theorem 2.1] Let X, Y and Z be three Banach spaces.

(i) If the set $\Phi^{b}(Y, Z)$ is not empty, then

$$
\begin{aligned}
& F \in \mathcal{F}_{+}^{b}(X, Y), T \in \mathcal{L}(Y, Z) \text { imply } T F \in \mathcal{F}_{+}^{b}(X, Z) . \\
& F \in \mathcal{F}_{-}^{b}(X, Y), T \in \mathcal{L}(Y, Z) \text { imply } T F \in \mathcal{F}_{-}^{b}(X, Z) \text {. }
\end{aligned}
$$


(ii) If the set $\Phi^{b}(X, Y)$ is not empty, then

$$
\begin{array}{lll}
F \in \mathcal{F}_{+}^{b}(Y, Z), T \in \mathcal{L}(X, Y) & \text { imply } & F T \in \mathcal{F}_{+}^{b}(X, Z) . \\
F \in \mathcal{F}_{-}^{b}(Y, Z), T \in \mathcal{L}(X, Y) & \text { imply } & F T \in \mathcal{F}_{-}^{b}(X, Z) .
\end{array}
$$

Proposition $2.2[9,10,27]$

(i) $\Phi_{+T}, \Phi_{-T}$ and $\Phi_{T}$ are open.

(ii) $i(\lambda-T)$ is constant on any component of $\Phi_{T}$.

(iii) $\quad \alpha(\lambda-T)$ and $\beta(\lambda-T)$ are constant on any component of $\Phi_{T}$ except on a discrete set of points at which they have larger values.

Remark 2.1 Let $A \in \mathcal{C}(X)$, then it follows from the closedness of $A$ that $\mathcal{D}(A)$ endowed with the graph norm $\|\cdot\|_{A}$ (i.e., $\left.\|\cdot\|_{A}:=\|x\|+\|A x\|\right)$ is a Banach space. Let $X_{A}$ denote $\left(\mathcal{D}(A),\|\cdot\|_{A}\right)$. In this new space the operator $A$ satisfies $\|A x\| \leq\|x\|_{A}$ and consequently $A$ is a bounded operator from $X_{A}$ into $X$. If $\hat{A}$ denotes the restriction of $A$ to $\mathcal{D}(A)$, we observe that $\alpha(\hat{A})=\alpha(A)$ and $\beta(\hat{A})=\beta(A)$.

Proposition 2.3 Let A be a closed operator in a complex Banach space $X$ with nonempty resolvent set. If $\Phi_{A}$ is connected, then

$$
\sigma_{e g}(A)=\sigma_{e a p}(A) \text { and } \sigma_{e w}(A)=\sigma_{e \delta}(A) .
$$

Proof It easy to check that $\sigma_{e g}(A) \subset \sigma_{e a p}(A)$. For the second inclusion we take $\mu \in \rho_{e g}(A)$, then $\mu \in \Phi_{+A}=\Phi_{A} \cup\left(\Phi_{+A} \backslash \Phi_{A}\right)$. Hence, we will discuss these two cases:

1st case If $\mu \in \Phi_{A}$ then $i(A-\mu)=0$.

Indeed, let $\mu_{0} \in \rho(A)$, then $\mu_{0} \in \Phi_{A}$ and $i\left(A-\mu_{0}\right)=0$. It follows from Proposition 2.2 (ii) that $i(A-\mu)$ is constant on any component of $\Phi_{A}$, therefore $\rho(A) \subseteq \Phi_{A}$, then $i(A-\mu)=0$ for all $\mu \in \Phi_{A}$. This shows that $\mu \in \rho_{\text {eap }}(A)$.

2nd case If $\mu \in\left(\Phi_{+A} \backslash \Phi_{A}\right)$, then

$$
\alpha(A-\mu)<\infty \text { and } \beta(A-\mu)=+\infty .
$$

So, $i(A-\mu)=-\infty<0$.

Hence, we obtain the second inclusion from the above two cases. Reasoning in the same way, we get the second equality.

Lemma 2.1 [18, Lemma 1] Let A be a closed operator in a complex Banach space $X$ with nonempty resolvent set. For $\lambda, \mu \in \rho_{b}(A)$, we have the resolvent identity

$$
R_{b}(A, \lambda)-R_{b}(A, \mu)=(\lambda-\mu) R_{b}(A, \lambda) R_{b}(A, \mu)+R_{b}(A, \lambda) S(\lambda, \mu) R_{b}(A, \mu),
$$

where $S(.$, .) is a finite rank operator with the following expression

$$
S(\lambda, \mu):=\left[(A-(\lambda+1)) P_{\lambda}-(A-(\mu+1)) P_{\mu}\right] .
$$

Lemma 2.2 [18, Lemma 2] Let $X$ and $Y$ be two complex Banach space, $B: Y \longrightarrow X$ and $C: X \longrightarrow Y$ linear operators. Then,

(i) $R_{b}(A, \mu) B$ is closable for some $\mu \in \rho_{b}(A)$ if and only if it is closable for all such $\mu$.

(ii) $C$ is $A$-bounded if and only if $C R_{b}(A, \mu)$ is bounded for some (or every) $\mu \in \rho_{b}(A)$.

(iii) If $B$ and $C$ satisfy the conditions ( $i)$ and (ii), respectively, and $B$ is densely defined, then $C R_{b}(A, \lambda) S(\lambda, \mu) R_{b}(A, \mu), \overline{R_{b}(A, \lambda) S(\lambda, \mu) R_{b}(A, \mu) B}$ and $\bar{C} R_{b}(A, \lambda) S(\lambda, \mu) R_{b}(A, \mu) B$ are operators of finite rank for any $\lambda, \mu \in \rho_{b}(A)$. 
Definition 2.2 Let $X$ and $Y$ be two Banach spaces. An operator $A \in \mathcal{L}(X, Y)$ is said to be weakly compact if $A(B)$ is relatively weakly compact in $Y$ for every bounded subset $B \subset X$.

The family of weakly compact operators from $X$ into $Y$ is denoted by $\mathcal{W}(X, Y)$. If $X=Y$ the family of weakly compact operators on $X, \mathcal{W}(X):=\mathcal{W}(X, X)$ is a closed two-sided ideal of $\mathcal{L}(X)$ containing $\mathcal{K}(X)$ (see $[8,11])$.

Definition 2.3 A Banach space $X$ is said to have the Dunford-Pettis property (for short property DP) if for each Banach space $Y$ every weakly compact operator $T: X \longrightarrow Y$ takes weakly compact sets in $X$ into norm compact sets of $Y$. For example it is well known that any $L_{1}$-space has the property DP [7].

Definition 2.4 Let $X$ be a Banach space. An operator $S \in \mathcal{L}(X)$ is called strictly singular if, for every infinite-dimensional subspace $M$ of $X$, the restriction of $S$ to $M$ is not a homeomorphism.

Let $\mathcal{S}(X)$ denote the set of strictly singular operators on $X$.

The concept of strictly singular operators was introduced in the pioneering paper by Kato [17] as a generalization of the notion of compact operators. For a detailed study of the properties of strictly singular operators we refer to [11,17]. Note that $\mathcal{S}(X)$ is a closed two-sided ideal of $\mathcal{L}(X)$ containing $\mathcal{K}(X)$. If $X$ is a Hilbert space then $\mathcal{S}(X)=\mathcal{K}(X)$. The class of weakly compact operators in $L_{1}$-spaces (resp. $\mathcal{C}(\Omega)$-spaces with $\Omega$ a compact Haussdorff space) is nothing else than the family of strictly singular operators on $L_{1}$-spaces (resp. $\mathcal{C}(\Omega)$-spaces) (see [23, Theorem 1]).

Let $X$ be a Banach space. If $N$ is a closed subspace of $X$, we denote by $\pi_{N}$ the quotient map $X \longrightarrow X / N$. The codimension of $N, \operatorname{codim}(N)$, is defined to be the dimension of the vector space $X / N$.

Definition 2.5 Let $X$ be a Banach space. An operator $S \in \mathcal{L}(X)$ is said to be strictly cosingular if there exists no closed subspace $N$ of $X$ with $\operatorname{codim}(N)=\infty$ such that $\pi_{N} S: X \longrightarrow X / N$ is surjective.

Let $\mathcal{C S}(X)$ denote the set of strictly cosingular operators on $X$. This class of operators was introduced by Pelczynski [23], it forms a closed two-sided ideal of $\mathcal{L}(X)$ [31].

Definition 2.6 We say that a Banach space $X$ is weakly compact generating (w.c.g.) if the linear span of some weakly compact subset is dense in $X$. For more details and results see [6]. In particular, all separable and all reflexive Banach spaces are w.c.g. as well as $L_{1}(\Omega, d \mu)$ if $(\Omega, \mu)$ is $\sigma$-finite. It is proved in [32] that if $X$ is a w.c.g., then

$$
\mathcal{F}_{+}(X)=\mathcal{S}(X) \text { and } \mathcal{F}_{-}(X)=\mathcal{C S}(X) .
$$

Remark 2.2 Let $(\Omega, \Sigma, \mu)$ be a positive measure space and let $X_{p}$ denote the spaces $L_{p}(\Omega, d \mu)$ with $1 \leq p<\infty$. Since the spaces $X_{p}, 1 \leq p<\infty$, are w.c.g., then we can deduce from what precedes that

$$
\mathcal{K}\left(X_{p}\right) \subset \mathcal{F}_{+}\left(X_{p}\right) \cap \mathcal{F}_{-}\left(X_{p}\right) .
$$

Definition 2.7 We say that $X$ is subprojective if given any closed infinite dimensional subspace $M$ of $X$, there exists a closed infinite dimensional subspace $N$ contained in $M$ and a continuous projection from $X$ onto $N$. For example, the space $L_{p}(2 \leq p<\infty)$ is subprojective [33]. 
Definition 2.8 We say that $X$ is superprojective if every subspace $V$ having infinite codimension in $X$ is contained in a closed subspace $W$ having infinite codimension in $X$ as it exists a bounded projection from $X$ to $W$. For example, the spaces $L_{p}(1<p \leq 2)$ are superprojective [33].

Let $X$ be a w.c.g Banach space. It is proved in [29] that if $X$ is superprojective (resp. subprojective), then $\mathcal{S}(X) \subset \mathcal{C S}(X)$ (resp. $\mathcal{C S}(X) \subset \mathcal{S}(X)$ ). Accordingly, we have the following result:

Proposition 2.4 Let $X$ be a w.c.g Banach space, then

(i) If $X$ is superprojective, then $\mathcal{S}(X) \subset \mathcal{F}_{+}(X) \cap \mathcal{F}_{-}(X)$.

(ii) If $X$ is subprojective, then $\mathcal{C S}(X) \subset \mathcal{F}_{+}(X) \cap \mathcal{F}_{-}(X)$.

\section{The main result}

Let $X, Y$ and $Z$ be three Banach spaces. In this paper, we consider the linear operators $\Gamma_{X}$ from $X$ into $Z$ and $\Gamma_{Y}$ from $Y$ into $Z$, therefore we define in the Banach space $X \times Y$ the operator $\mathcal{A}_{0}$ as follows:

$$
\mathcal{A}_{0}:=\left(\begin{array}{cc}
A & B \\
C & D
\end{array}\right)
$$

$$
\mathcal{D}\left(\mathcal{A}_{0}\right):=\left\{\left(\begin{array}{l}
x \\
y
\end{array}\right) \text { such that } x \in \mathcal{D}(A), y \in \mathcal{D}(D) \cap \mathcal{D}(B) \text { and } \Gamma_{X} x=\Gamma_{Y} y\right\} .
$$

In what follows, we will assume that the following conditions hold:

(H1) The operator $A$ is densely defined and closable.

It follows from Remark 2.1 that $\mathcal{D}(\bar{A})$, the domain of closure $\bar{A}$ of $A$, coincides with the Banach space $X_{A}$ which is contained in $X$.

(H2) $\mathcal{D}(A) \subset \mathcal{D}\left(\Gamma_{X}\right) \subset X_{A}$ and $\Gamma_{X}$ is bounded as a mapping from $X_{A}$ into $Z$.

(H3) The set $\mathcal{D}(A) \cap \mathcal{N}\left(\Gamma_{X}\right)$ is dense in $X$ and the resolvent set of the restriction $A_{1}:=\left.A\right|_{\mathcal{D}(A) \cap \mathcal{N}\left(\Gamma_{X}\right)}$ is not empty, i.e. $\rho\left(A_{1}\right) \neq \emptyset$.

(H4) $\mathcal{D}(A) \subset \mathcal{D}(C) \subset X_{A}$ and $C$ is $A_{1}$-bounded.

Remark 3.1 It follows from (H3) that $A_{1}$ is a closed operator in the Banach space $X_{A}$ with nonempty resolvent set. For $\lambda \in \rho_{b}\left(A_{1}\right)$, let $P_{\lambda}$ denotes the corresponding finite rank Riesz projector with range and kernel denoted by $\mathcal{R}_{\lambda}$ and $\mathcal{N}_{\lambda}$, respectively.

Let $A_{1 \lambda}$ the operator defined by:

$$
A_{1 \lambda}=\left(A_{1}-\lambda\right)\left(I-P_{\lambda}\right)+P_{\lambda}
$$

because $\mathcal{D}\left(A_{1}\right)$ is $P_{\lambda}$-invariant, $A_{1 \lambda}$ has the same domain of $A_{1}$ with respect to the decomposition $X=\mathcal{R}_{\lambda} \oplus \mathcal{N}_{\lambda}$, we can write $A_{1 \lambda}=\left(A_{1}-\left.\lambda\right|_{\mathcal{N}_{\lambda}}\right) \oplus I$. Since $\sigma\left(A_{1}-\left.\lambda\right|_{\mathcal{N}_{\lambda}}\right)=$ $\sigma\left(A_{1}-\lambda\right) \backslash\{0\}, A_{1 \lambda}$ has bounded inverse denoted by $R_{b}\left(A_{1}, \lambda\right)$ and called the Browder resolvent. This clearly extends the usual resolvent $\left(A_{1}-\lambda\right)^{-1}$ from $\rho\left(A_{1}\right)$ to $\rho_{b}\left(A_{1}\right)$.

Lemma 3.1 Under the assumptions $(H 1)-(H 3)$, for any $\lambda \in \rho_{b}\left(A_{1}\right)$, the following decomposition holds:

$$
\mathcal{D}(A)=\mathcal{D}\left(A_{1}\right) \oplus \mathcal{N}\left(A_{\lambda}\right),
$$

where $A_{\lambda}$ is the operator defined on $\mathcal{D}(A)$ by: $A_{\lambda}:=(A-\lambda)\left(I-P_{\lambda}\right)+P_{\lambda}$. 
Proof Let $\lambda \in \rho_{b}\left(A_{1}\right)$. It is clear that the sum (3.1) is contained in $\mathcal{D}(A)$ and it follows that

$$
\mathcal{D}\left(A_{1}\right) \cap \mathcal{N}\left(A_{\lambda}\right)=\mathcal{N}\left(A_{1 \lambda}\right) .
$$

Since the operator $A_{1 \lambda}$ is invertible, then $\mathcal{N}\left(A_{1 \lambda}\right)=\{0\}$ and we get

$$
\mathcal{D}\left(A_{1}\right) \cap \mathcal{N}\left(A_{\lambda}\right)=\{0\} .
$$

For any $f \in \mathcal{D}(A)$, we set

$$
g=R_{b}\left(A_{1}, \lambda\right) A_{\lambda} f \in \mathcal{D}\left(A_{1}\right) .
$$

Then, $f-g \in \mathcal{N}\left(A_{\lambda}\right)$ and $f=g+f-g \in \mathcal{D}\left(A_{1}\right)+\mathcal{N}\left(A_{\lambda}\right)$.

Lemma 3.2 Under the assumptions $(H 1)-(H 3)$, for any $\lambda \in \rho_{b}\left(A_{1}\right)$, the restriction

$$
\Gamma_{\lambda}:=\left.\Gamma_{X}\right|_{\mathcal{N}}\left(A_{\lambda}\right)
$$

is injective and

$$
\mathcal{R}\left(\Gamma_{\lambda}\right)=\Gamma_{X}\left(\mathcal{N}\left(A_{\lambda}\right)\right)=\Gamma_{X}(\mathcal{D}(A)):=Z_{1}
$$

does not depend on $\lambda$.

Proof Let $\lambda \in \rho_{b}\left(A_{1}\right)$. The injectivity of the operator $\Gamma_{\lambda}$ follows from the fact that:

$$
\mathcal{N}\left(\Gamma_{\lambda}\right):=\mathcal{N}\left(A_{\lambda}\right) \cap \mathcal{N}\left(\Gamma_{X}\right)=\mathcal{N}\left(A_{1 \lambda}\right)=\{0\} .
$$

It follows from the definition of the operator $\Gamma_{\lambda}$ that his range coincides with $\Gamma_{X}\left(\mathcal{N}\left(A_{\lambda}\right)\right)$. Therefore, it follows from $(H 3)$ that $\Gamma_{X}\left(\mathcal{D}\left(A_{1}\right)\right)=\{0\}$. Hence, the use of Lemma 3.1 and the linearity of the operator $\Gamma_{X}$ make us conclude that $\Gamma_{X}\left(\mathcal{N}\left(A_{\lambda}\right)\right)=\Gamma_{X}(\mathcal{D}(A))$. Hence $\mathcal{R}\left(\Gamma_{\lambda}\right)$ does not depend on $\lambda$.

In the following, for $\lambda \in \rho_{b}\left(A_{1}\right)$, the inverse $K_{\lambda}$ of the operator $\Gamma_{\lambda}$ will play an important role:

$$
K_{\lambda}:=\left(\left.\Gamma_{X}\right|_{\mathcal{N}\left(A_{\lambda}\right)}\right)^{-1}: Z_{1} \longrightarrow \mathcal{N}\left(A_{\lambda}\right) \subset X .
$$

In the other words, $K_{\lambda} z=x$ means that $x \in \mathcal{D}(A)$ and

$$
\begin{aligned}
& A_{\lambda} x=0, \\
& \Gamma_{X} x=z .
\end{aligned}
$$

Lemma 3.3 If $\lambda_{1}, \lambda_{2} \in \rho_{b}\left(A_{1}\right)$, then

$$
K_{\lambda_{1}}-K_{\lambda_{2}}=R_{b}\left(A_{1}, \lambda_{1}\right)\left[\left(\lambda_{1}-\lambda_{2}\right)+S\left(\lambda_{1}, \lambda_{2}\right)\right] K_{\lambda_{2}},
$$

where $S$ is the finite rank operator defined by

$$
S\left(\lambda_{1}, \lambda_{2}\right):=\left[\left(A_{1}-\left(\lambda_{1}+1\right)\right) P_{\lambda_{1}}-\left(A_{1}-\left(\lambda_{2}+1\right)\right) P_{\lambda_{2}}\right] .
$$

If $K_{\lambda}$ is closable for at least one $\lambda \in \rho_{b}\left(A_{1}\right)$, then it is closable for all such $\lambda$, and the above relation holds with $K_{\lambda_{j}}$ replaced by the closures $\bar{K}_{\lambda_{j}}, j=1,2$. 
Proof Let $z \in Z_{1}$ and set $x=x_{1}-x_{2}$ where $x_{j}=K_{\lambda_{j}} z, j=1,2$. The use of Eq. (3.4) shows that

$$
\begin{aligned}
A_{\lambda_{1} x} & =-A_{\lambda_{1}} x_{2} \\
& =-\left[\left(A-\lambda_{1}\right)\left(I-P_{\lambda_{1}}\right)+P_{\lambda_{1}}\right] x_{2} \\
& =-\left[\left(A-\lambda_{2}\right)\left(I-P_{\lambda_{1}}\right)+\left(\lambda_{2}-\lambda_{1}\right)\left(I-P_{\lambda_{1}}\right)+P_{\lambda_{1}}\right] x_{2} \\
& =\left[\left(A-\left(\lambda_{1}+1\right)\right) P_{\lambda_{1}}-\left(A-\left(\lambda_{2}+1\right)\right) P_{\lambda_{2}}+\left(\lambda_{1}-\lambda_{2}\right)\right] x_{2} \\
& =\left[\left(A_{1}-\left(\lambda_{1}+1\right)\right) P_{\lambda_{1}}-\left(A_{1}-\left(\lambda_{2}+1\right)\right) P_{\lambda_{2}}+\left(\lambda_{1}-\lambda_{2}\right)\right] x_{2} \\
& =\left[\left(\lambda_{1}-\lambda_{2}\right)+S\left(\lambda_{1}, \lambda_{2}\right)\right] x_{2} .
\end{aligned}
$$

Therefore, it follows from Eq. (3.5) that $\Gamma_{X} x=\Gamma_{X} x_{1}-\Gamma_{X} x_{2}=0$. Hence $x \in \mathcal{D}\left(A_{1}\right)$ and $x=R_{b}\left(A_{1}, \lambda_{1}\right)\left[\left(\lambda_{1}-\lambda_{2}\right)+S\left(\lambda_{1}, \lambda_{2}\right)\right] x_{2}$. This allowed us to conclude that

$$
K_{\lambda_{1}}-K_{\lambda_{2}}=R_{b}\left(A_{1}, \lambda_{1}\right)\left[\left(\lambda_{1}-\lambda_{2}\right)+S\left(\lambda_{1}, \lambda_{2}\right)\right] K_{\lambda_{2}} .
$$

So

$$
K_{\lambda_{2}}-K_{\lambda_{1}}=-R_{b}\left(A_{1}, \lambda_{2}\right)\left[\left(\lambda_{1}-\lambda_{2}\right)+S\left(\lambda_{1}, \lambda_{2}\right)\right] K_{\lambda_{1}}
$$

Hence

$$
\left[\left(\lambda_{1}-\lambda_{2}\right)+S\left(\lambda_{1}, \lambda_{2}\right)\right] K_{\lambda_{1}}=A_{1 \lambda_{2}} R_{b}\left(A_{1}, \lambda_{1}\right)\left[\left(\lambda_{1}-\lambda_{2}\right)+S\left(\lambda_{1}, \lambda_{2}\right)\right] K_{\lambda_{2}} .
$$

Since the operator $S\left(.\right.$, .) is of finite rank and $A_{1 \lambda_{2}} R_{b}\left(A_{1}, \lambda_{1}\right)$ is bounded and boundedly invertible, $K_{\lambda_{1}}$ is closable if $K_{\lambda_{2}}$ is such, in which case their closures $\bar{K}_{\lambda_{j}}, j=1,2$ satisfy the same relations.

Concerning the operators $K_{\lambda}, D, \Gamma_{Y}$ and $B$ we impose the following conditions:

(H5) For some (hence for all) $\lambda \in \rho_{b}\left(A_{1}\right)$, the operator $K_{\lambda}$ is bounded as a mapping from $Z$ into $X$.

(H6) The operator $D$ is densely defined and closed.

(H7) $\mathcal{D}\left(\Gamma_{Y}\right) \supset \mathcal{D}(D) \cap \mathcal{D}(B)$, the set

$$
Y_{1}=\left\{y \text { such that } y \in \mathcal{D}(D) \cap \mathcal{D}(B) \text { and } \Gamma_{Y} y \in Z_{1}\right\}
$$

is dense in $Y$ and the restriction of $\Gamma_{Y}$ to this set is bounded as an operator from $Y$ into $Z$.

(H8) For some (and hence for all, see Lemma $2.2(i)) \lambda \in \rho_{b}\left(A_{1}\right)$, the operator $R_{b}\left(A_{1}, \lambda\right) B$ is closable and its closure $\overline{R_{b}\left(A_{1}, \lambda\right) B}$ is bounded.

Remark 3.2 We will denote by

(i) $\bar{\Gamma}_{X}$ the extension of $\Gamma_{X}$ by continuity to $X_{A}=\mathcal{D}(\bar{A})$. It is a bounded operator from $X_{A}$ into $Z$.

(ii) $\bar{\Gamma}_{Y}^{0}$ the extension of $\left.\Gamma_{Y}\right|_{Y_{1}}$ by continuity to all of $Y$.

(iii) $\bar{K}_{\lambda}$ the extension of $K_{\lambda}$ to the closure $\bar{Z}_{1}$ of $Z_{1}$ with respect to the norm of $Z$. Without loss of generality we assume that $\bar{Z}_{1}=Z$.

We can easy verify that the operator $\bar{K}_{\lambda}$ is also bounded as a mapping from $\bar{Z}_{1}$ to $X_{A}$.

In the space $Y$, for $\lambda \in \rho_{b}\left(A_{1}\right)$, we consider the operator

$$
M_{\lambda}:=D+C K_{\lambda} \Gamma_{Y}-C_{\lambda} B,
$$

where $C_{\lambda}:=C R_{b}\left(A_{1}, \lambda\right)$. The operator $M_{\lambda}$ is defined on the set $Y_{1}$, which is dense in $Y$ according to $(H 7)$. 
Remark 3.3 For any $\lambda_{1}$ and $\lambda_{2} \in \rho_{b}\left(A_{1}\right)$, it follows from the resolvent identity that:

$$
M_{\lambda_{1}}-M_{\lambda_{2}}=C_{\lambda_{1}}\left[\left(\lambda_{2}-\lambda_{1}\right)-S\left(\lambda_{1}, \lambda_{2}\right)\right]\left[-K_{\lambda_{2}} \Gamma_{Y}+R_{b}\left(A_{1}, \lambda_{2}\right) B\right] .
$$

It follows, immediately, from Lemma 2.2 (ii) that $C_{\lambda}$ is bounded. Therefore we observe that $\Gamma_{Y}$ is bounded on this domain by assumption (H7), that $K_{\lambda}$ is bounded by assumption (H5), that $\mathcal{R}\left(K_{\lambda}\right) \subset \mathcal{D}(A) \subset \mathcal{D}(C)$ and finally $S$ is of finite rank. Now using (H8) we infer that if $M_{\lambda}$ is closable as an operator in $Y$ for some $\lambda \in \rho_{b}\left(A_{1}\right)$, then it is closable for all $\lambda \in \rho_{b}\left(A_{1}\right)$.

We emphasize also that the domain of $\bar{M}_{\lambda}$ does not depend on $\lambda$. Indeed the difference

$$
\bar{M}_{\lambda_{1}}-\bar{M}_{\lambda_{2}}=C_{\lambda_{1}}\left[\left(\lambda_{2}-\lambda_{1}\right)-S\left(\lambda_{1}, \lambda_{2}\right)\right]\left[-\bar{K}_{\lambda_{2}} \bar{\Gamma}_{Y}^{0}+\bar{R}_{b}\left(A_{1}, \lambda_{2}\right) B\right]
$$

is a bounded operator.

Lemma 3.4 For $\lambda \in \rho_{b}\left(A_{1}\right)$ and $x \in \mathcal{D}(A)$ we have

$$
A_{\lambda} x=A_{1 \lambda}\left(I-K_{\lambda} \Gamma_{X}\right) x
$$

and the operator $I-K_{\lambda} \Gamma_{X}$ is the projection from $\mathcal{D}\left(A_{1}\right)$ parallel to $\mathcal{N}\left(A_{\lambda}\right)$.

Proof Let $x \in \mathcal{D}(A)$, then we have

$$
x=\left(I-K_{\lambda} \Gamma_{X}\right) x+K_{\lambda} \Gamma_{X} x .
$$

The first summand belongs to $\mathcal{D}\left(A_{1}\right)$ because $x_{1}=\left(I-K_{\lambda} \Gamma_{X}\right) x \in \mathcal{D}(A)$ and $\Gamma_{X} x_{1}=$ $\Gamma_{X} x-\Gamma_{X} K_{\lambda} \Gamma_{X} x=0$. Therefore, it is clear that the second summand belongs to $\mathcal{N}\left(A_{\lambda}\right)$. Now, we apply Lemma 3.1 to get the result.

For each $\lambda \in \rho_{b}\left(A_{1}\right)$, we define the bounded, lower and upper triangular operator matrices

$$
\mathbb{T}_{1}(\lambda)=\left(\begin{array}{cc}
I & 0 \\
C_{\lambda} & I
\end{array}\right), \quad \mathbb{T}_{2}(\lambda)=\left(\begin{array}{cc}
I & -\bar{K}_{\lambda} \bar{\Gamma}_{Y}^{0}+\overline{R_{b}\left(A_{1}, \lambda\right) B} \\
0 & I
\end{array}\right),
$$

the finite rank operator-matrix

$$
\mathbb{N}(\lambda)=\left(\begin{array}{cc}
{[A-(\lambda+1)] P_{\lambda}} & 0 \\
0 & 0
\end{array}\right)
$$

and the diagonal operator-matrix

$$
\mathbb{D}_{0}(\lambda)=\left(\begin{array}{cc}
A_{1 \lambda} & 0 \\
0 & M_{\lambda}-\lambda I
\end{array}\right)
$$

with domain $\mathcal{D}\left(A_{1}\right) \times Y_{1}$.

Theorem 3.1 Assume that the conditions (H1)-(H8) are satisfied. Then $\mathcal{A}_{0}$ is closable in $X \times Y$ if only if, the operator $M_{\lambda}:=D+C K_{\lambda} \Gamma_{Y}-C_{\lambda} B$ is closable for some $\lambda \in \rho_{b}\left(A_{1}\right)$, or equivalently, for all $\lambda \in \rho_{b}\left(A_{1}\right)$. Moreover, the closure $\mathcal{A}$ of $\mathcal{A}_{0}$ is given by the relation

$$
\mathcal{A}:=\overline{\mathcal{A}_{0}}=\lambda I+\mathbb{T}_{1}(\lambda) \mathbb{D}(\lambda) \mathbb{T}_{2}(\lambda)+\mathbb{N}(\lambda),
$$

where $\mathbb{D}(\lambda):=\overline{\mathbb{D}_{0}(\lambda)}=\left(\begin{array}{cc}A_{1 \lambda} & 0 \\ 0 & \overline{M_{\lambda}}-\lambda I\end{array}\right)$ with domain $\mathcal{D}\left(A_{1}\right) \times \mathcal{D}\left(\overline{M_{\lambda}}\right)$. 
Proof Let $\lambda \in \rho_{b}\left(A_{1}\right)$. We shall show that $\mathcal{A}_{0}-\lambda I=\mathcal{G}_{\lambda}$ where

$$
\begin{aligned}
\mathcal{G}_{\lambda}= & \left(\begin{array}{cc}
I & 0 \\
C_{\lambda} & I
\end{array}\right)\left(\begin{array}{cc}
A_{1 \lambda} & 0 \\
0 & M_{\lambda}-\lambda I
\end{array}\right)\left(\begin{array}{cc}
I & -K_{\lambda} \Gamma_{Y}+R_{b}\left(A_{1}, \lambda\right) B \\
0 & I
\end{array}\right) \\
& +\left(\begin{array}{cc}
{[A-(\lambda+1)] P_{\lambda}} & 0 \\
0 & 0
\end{array}\right) .
\end{aligned}
$$

To get this equality, we will prove that $\mathcal{D}\left(\mathcal{G}_{\lambda}\right) \subset \mathcal{D}\left(\mathcal{A}_{0}\right)$ and $\mathcal{A}_{0}-\lambda I=\mathcal{G}_{\lambda}$.

First, it follows that $\mathcal{D}\left(\mathcal{G}_{\lambda}\right)$ consists of the elements of the form

$$
\left(\begin{array}{l}
x \\
y
\end{array}\right)=\left(\begin{array}{c}
x^{\prime}-K_{\lambda} \Gamma_{Y} y+R_{b}\left(A_{1}, \lambda\right) B y \\
y
\end{array}\right),
$$

where $x^{\prime}$ and $y$ run through $\mathcal{D}\left(A_{1}\right)=\mathcal{D}(A) \cap \mathcal{N}\left(\Gamma_{X}\right)$ and $\mathcal{D}\left(M_{\lambda}\right)$, respectively. Therefore $x \in \mathcal{D}(A), y \in \mathcal{D}(D) \cap \mathcal{D}(B)$ and $\Gamma_{X} x=\Gamma_{X}\left(K_{\lambda} \Gamma_{Y} y\right)=\Gamma_{Y} y$. Hence

$$
\left(\begin{array}{l}
x \\
y
\end{array}\right) \in \mathcal{D}\left(\mathcal{A}_{0}\right)
$$

and

$$
\mathcal{D}\left(\mathcal{G}_{\lambda}\right) \subset \mathcal{D}\left(\mathcal{A}_{0}\right)
$$

Second, let

$$
\left(\begin{array}{l}
x \\
y
\end{array}\right) \in \mathcal{D}\left(\mathcal{A}_{0}\right)
$$

i.e. $x \in \mathcal{D}(A), y \in \mathcal{D}(D) \cap \mathcal{D}(B)$ and $\Gamma_{X} x=\Gamma_{Y} y$.

$$
\mathcal{G}_{\lambda}\left(\begin{array}{l}
x \\
y
\end{array}\right)=\left(\begin{array}{cc}
A_{1 \lambda} & 0 \\
C & M_{\lambda}-\lambda I
\end{array}\right)\left(\begin{array}{c}
\left(I-K_{\lambda} \Gamma_{X}\right) x+R_{b}\left(A_{1}, \lambda\right) B y \\
y
\end{array}\right)+\left(\begin{array}{c}
{[A-(\lambda+1)] P_{\lambda} x} \\
0
\end{array}\right)
$$

Using Lemma 3.4, we get

$$
\begin{aligned}
\mathcal{G}_{\lambda}\left(\begin{array}{l}
x \\
y
\end{array}\right) & =\left(\begin{array}{c}
A_{\lambda} x+B y \\
C\left(x-K_{\lambda} \Gamma_{X} x+R_{b}\left(A_{1}, \lambda\right) B y\right)+\left(M_{\lambda}-\lambda I\right) y
\end{array}\right)+\left(\begin{array}{c}
{[A-(\lambda+1)] P_{\lambda} x} \\
0
\end{array}\right) \\
& =\left(\begin{array}{c}
(A-\lambda I) x+B y \\
C x+(D-\lambda I) y
\end{array}\right)=\left(\mathcal{A}_{0}-\lambda I\right)\left(\begin{array}{l}
x \\
y
\end{array}\right),
\end{aligned}
$$

therefore $\mathcal{A}_{0}-\lambda I=\mathcal{G}_{\lambda}$.

Finally, it is easy to check that $\mathbb{T}_{1}(\lambda)$ and $\mathbb{T}_{2}(\lambda)$ are bounded and have bounded inverses. Then we deduce from the factorization of $\mathcal{A}_{0}-\lambda I$ that $\mathcal{A}_{0}$ is closable in $X \times Y$ if only if $M_{\lambda}$ is closable as a mapping in $Y$. Moreover, if $M_{\lambda}$ is closable and $\bar{M}_{\lambda}$ denotes its closure, then for the closure $\mathcal{A}$ of $\mathcal{A}_{0}$ we get

$$
\mathcal{A}:=\overline{\mathcal{A}_{0}}=\lambda I+\mathbb{T}_{1}(\lambda)\left(\begin{array}{cc}
A_{1 \lambda} & 0 \\
0 & \overline{M_{\lambda}}-\lambda I
\end{array}\right) \mathbb{T}_{2}(\lambda)+\mathbb{N}(\lambda) .
$$


Lemma 3.5 (i) If $C_{\lambda} \in \mathcal{F}_{+}^{b}(X, Y)$ for some $\lambda \in \rho_{b}\left(A_{1}\right)$, then $C_{\lambda} \in \mathcal{F}_{+}^{b}(X, Y)$ for all $\lambda \in \rho_{b}\left(A_{1}\right)$, and $\sigma_{\text {eap }}\left(\overline{M_{\lambda}}\right)$ does not depend on the choice of $\lambda$.

(ii) If $C_{\lambda} \in \mathcal{F}_{-}^{b}(X, Y)$ for some $\lambda \in \rho_{b}\left(A_{1}\right)$, then $C_{\lambda} \in \mathcal{F}_{-}^{b}(X, Y)$ for all $\lambda \in \rho_{b}\left(A_{1}\right)$, and $\sigma_{e \delta}\left(\overline{M_{\lambda}}\right)$ does not depend on the choice of $\lambda$.

Proof (i) Let $\lambda_{0} \in \rho_{b}\left(A_{1}\right)$ such that $C_{\lambda_{0}} \in \mathcal{F}_{+}^{b}(X, Y)$. From the resolvent identity we have,

$$
C_{\lambda}-C_{\lambda_{0}}=C_{\lambda_{0}}\left[\left(\lambda-\lambda_{0}\right)+S\left(\lambda, \lambda_{0}\right)\right] R_{b}\left(A_{1}, \lambda\right)
$$

for all $\lambda \in \rho_{b}\left(A_{1}\right)$. Thus writing $C_{\lambda}$ in the form

$$
C_{\lambda}=C_{\lambda_{0}}\left[I+\left(\left(\lambda-\lambda_{0}\right)+S\left(\lambda, \lambda_{0}\right)\right) R_{b}\left(A_{1}, \lambda\right)\right]
$$

and using Proposition 2.1(ii) we deduce that $C_{\lambda} \in \mathcal{F}_{+}^{b}(X, Y)$ and the difference

$$
\bar{M}_{\lambda}-\bar{M}_{\lambda_{0}}=C_{\lambda}\left[\left(\lambda_{0}-\lambda\right)-S\left(\lambda, \lambda_{0}\right)\right]\left[-\bar{K}_{\lambda_{0}} \bar{\Gamma}_{Y}^{0}+\overline{R_{b}\left(A_{1}, \lambda_{0}\right) B}\right]
$$

is in $\mathcal{F}_{+}^{b}(Y, Y)$. Now, the use of Theorem 3.1(i) and Remark 3.3 in [20] make us conclude that $\sigma_{\text {eap }}\left(\overline{M_{\lambda}}\right)$ does not depend on the choice of $\lambda$.

(ii) This assertion can be proved in the same way as $(i)$.

We are now in the position to express the main results of this section.

Theorem 3.2 Let assumptions (H1)-(H8) hold, then

(i) If for some $\lambda \in \rho_{b}\left(A_{1}\right)$, the operator $C_{\lambda} \in \mathcal{F}_{+}^{b}(X, Y)$ then

$$
\sigma_{\text {eap }}(\mathcal{A}) \cap \rho_{b}\left(A_{1}\right)=\sigma_{\text {eap }}\left(\bar{M}_{\lambda}\right) \cap \rho_{b}\left(A_{1}\right) .
$$

(ii) If for some $\lambda \in \rho_{b}\left(A_{1}\right)$, the operator $C_{\lambda} \in \mathcal{F}_{-}^{b}(X, Y)$ then

$$
\sigma_{e \delta}(\mathcal{A}) \cap \rho_{b}\left(A_{1}\right)=\sigma_{e \delta}\left(\bar{M}_{\lambda}\right) \cap \rho_{b}\left(A_{1}\right) .
$$

Proof First, if $\mu \in \rho\left(A_{1}\right)$ then the relation in Eq. (3.7) became

$$
\mathcal{A}-\mu I:=\left(\begin{array}{cc}
I & 0 \\
C_{\mu} & I
\end{array}\right)\left(\begin{array}{cc}
A_{1}-\mu I & 0 \\
0 & \bar{M}_{\mu}-\mu I
\end{array}\right)\left(\begin{array}{cc}
I & -\bar{K}_{\mu} \bar{\Gamma}_{Y}^{0}+\overline{\left(A_{1}-\mu\right)^{-1} B} \\
0 & I
\end{array}\right) .
$$

It is clear that the external factors are bounded and have bounded inverses, therefore it follows from [27, Theorem 6.4] that $(\mathcal{A}-\mu I)$ is an upper semi-Fredholm operator if only if $\bar{M}_{\mu}-\mu I$ has this property furthermore the use of [22, Theorem 12, p. 152] and Remark 2.1 allow us to conclude that $i(\mathcal{A}-\mu I)=i\left(\bar{M}_{\mu}-\mu\right)$. Hence $\sigma_{\text {eap }}(\mathcal{A})=\sigma_{\text {eap }}\left(\bar{M}_{\mu}\right)$. Now, by Lemma 3.5 $(i)$, we deduce that $\sigma_{\text {eap }}(\mathcal{A})=\sigma_{\text {eap }}\left(\bar{M}_{\lambda}\right)$.

Second, if $\mu \in \sigma_{d}\left(A_{1}\right)$ then there exists $\varepsilon>0$ such that the disk

$$
\{\zeta \in \mathbb{C} \text { such that }|\zeta-\mu| \leq 2 \varepsilon\}
$$

does not contain points of $\sigma\left(A_{1}\right)$ different from $\mu$, and the Riesz projection $P_{\mu}$ of $A_{1}$ corresponding to $\mu$ is of finite rank. Consider the operator $\widetilde{A}_{1}:=A_{1}+\varepsilon P_{\mu}$. Then

$$
\{\lambda \in \mathbb{C} \text { such that } 0<|\lambda-\mu|<\varepsilon\} \subset \rho_{b}\left(A_{1}\right) \cap \rho_{b}\left(\widetilde{A}_{1}\right) \text {. }
$$

Until further notice we fix $\lambda \in \rho_{b}\left(A_{1}\right) \cap \rho_{b}\left(\widetilde{A}_{1}\right)$. 
We define the operator $\widetilde{\mathcal{A}}_{0}$ as the operator $\mathcal{A}_{0}$ but with $A$ replaced by $\widetilde{A}:=A+\varepsilon P_{\mu}$, so

$$
\widetilde{\mathcal{A}_{0}}=\left(\begin{array}{ll}
\widetilde{A} & B \\
C & D
\end{array}\right)=\mathcal{A}_{0}+\varepsilon\left(\begin{array}{cc}
P_{\mu} & 0 \\
0 & 0
\end{array}\right),
$$

and for the closure of $\widetilde{\mathcal{A}}_{0}$ we obtain

$$
\widetilde{\mathcal{A}}=\mathcal{A}+\varepsilon\left(\begin{array}{cc}
P_{\mu} & 0 \\
0 & 0
\end{array}\right) .
$$

It is clear that $\widetilde{\mathcal{A}}$ is a finite rank perturbation of $\mathcal{A}$. Therefore $\sigma_{\text {eap }}(\widetilde{\mathcal{A}})=\sigma_{\text {eap }}(\mathcal{A})$.

In the next, we will apply the obtained result of the first part of this proof on the operator $\widetilde{\mathcal{A}}$. So that, we consider the operator $\widetilde{M}_{\lambda}:=D+C \widetilde{K}_{\lambda} \Gamma_{X}-C R_{b}\left(\widetilde{A}_{1}, \lambda\right) B$ which is the perturbation of $M_{\lambda}$, for $\lambda \in \rho_{b}\left(\widetilde{A}_{1}\right)$. Here $\widetilde{K}_{\lambda}, \lambda \in \rho_{b}\left(\widetilde{A}_{1}\right)$, is the operator defined as $K_{\lambda}$ with $A$ replaced by $\widetilde{A}$. Hence, $\widetilde{K}_{\lambda} z=x$ means that $x \in \mathcal{D}(\widetilde{A}), \widetilde{A}_{\lambda} x=0$ and $\Gamma_{X} x=z$. Then, the operator $\widetilde{K}_{\lambda}$ is well defined for $\lambda \in \rho_{b}\left(\widetilde{A}_{1}\right)$.

The difference $\widetilde{K}_{\lambda}-K_{\lambda}$ is of finite rank. Indeed, take $z \in Z_{1}$ and put $\widetilde{u}=\widetilde{K}_{\lambda} z, u=K_{\lambda} z$, then $\tilde{u}-u$ satisfies the relations

$$
\Gamma_{X}(\widetilde{u}-u)=0 \text { and } A_{\lambda}(\widetilde{u}-u)=\left(\widetilde{A}_{\lambda}-\varepsilon P_{\mu}\right) \widetilde{u}=-\varepsilon P_{\mu} \widetilde{u} .
$$

This implies that $\widetilde{u}-u \in \mathcal{D}\left(A_{1}\right)$ and $\widetilde{u}-u=-\varepsilon R_{b}\left(A_{1}, \lambda\right) P_{\mu} \widetilde{u}$, so that

$$
\widetilde{K}_{\lambda}-K_{\lambda}=-\varepsilon P_{\mu} R_{b}\left(A_{1}, \lambda\right) \widetilde{K}_{\lambda}
$$

We can also see that the closure of the difference $C_{\lambda} B-C R_{b}\left(\widetilde{A}_{1}, \lambda\right) B$ is of finite rank. Indeed

$$
C_{\lambda} B-C R_{b}\left(\widetilde{A}_{1}, \lambda\right) B=-\varepsilon C_{\lambda} P_{\mu} R_{b}\left(\widetilde{A}_{1}, \lambda\right) B
$$

Using the two last results, we can easily check that the difference $\widetilde{M}_{\lambda}-M_{\lambda}$ is of finite rank. Since the operator $M_{\lambda}$ is closable in $Y$, we infer that its perturbation $\widetilde{M}_{\lambda}$ is closable in $Y$ as well, and we will denote its closure by $\widehat{M}_{\lambda}$. Since $\widehat{M}_{\lambda}-\bar{M}_{\lambda}$ is of finite rank, then $\sigma_{\text {eap }}\left(\widehat{M}_{\lambda}\right)=\sigma_{\text {eap }}\left(\bar{M}_{\lambda}\right)$.

Now, using the following relation

$$
C_{\lambda}-C R_{b}\left(\tilde{A}_{1}, \lambda\right)=-\varepsilon C_{\lambda} P_{\mu} R_{b}\left(\tilde{A}_{1}, \lambda\right)
$$

and the fact that for some $\lambda \in \rho_{b}\left(A_{1}\right)$, the operator $C_{\lambda} \in \mathcal{F}_{+}^{b}(X, Y)$, we can deduce easily that, for some $\lambda \in \rho_{b}\left(A_{1}\right), C R_{b}\left(\widetilde{A}_{1}, \lambda\right) \in \mathcal{F}_{+}^{b}(X, Y)$.

Hence, Lemma 3.5 implies that $\sigma_{e a p}\left(\widehat{M}_{\lambda}\right)$ is independent of $\lambda \in \rho_{b}\left(\widetilde{A}_{1}\right)$. Now, applying the first part of this proof for $\mu \in \rho\left(\widetilde{A}_{1}\right)$, we see that $\sigma_{\text {eap }}(\widetilde{\mathcal{A}})=\sigma_{\text {eap }}\left(\widehat{M}_{\mu}\right)$. Then, we get

$$
\sigma_{\text {eap }}(\mathcal{A})=\sigma_{\text {eap }}(\widetilde{\mathcal{A}})=\sigma_{\text {eap }}\left(\widehat{M}_{\mu}\right)=\sigma_{\text {eap }}\left(\widehat{M}_{\lambda}\right)=\sigma_{\text {eap }}\left(\bar{M}_{\lambda}\right)
$$

for any $\lambda \in \rho_{b}\left(A_{1}\right)$ as required, and the proof of $(i)$ is complete. A same reasoning allows us to reach the result $(i i)$.

Remark 3.4 It follows that Theorem 4.2 in [2] is obtained thanks to compacity of the operator $C\left(A_{1}-\lambda\right)^{-1}$ for $\lambda \in \rho\left(A_{1}\right)$. But here we extend this result for $\lambda \in \rho_{b}\left(A_{1}\right)$ and we give more generalization by assuming that $C R_{b}\left(A_{1}, \lambda\right)$ is in $\mathcal{F}_{+}^{b}(X, Y)$ or in $\mathcal{F}_{-}^{b}(X, Y)$. 
We will denote by $\mathbb{Q}(\lambda)$ the operator defined as follows

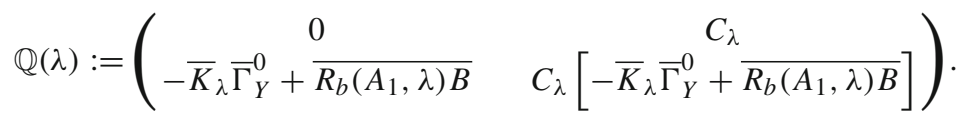

Theorem 3.3 Let assumptions (H1)-(H8) hold, then

(i) If for some $\lambda \in \rho_{b}\left(A_{1}\right)$, the operator $C_{\lambda} \in \mathcal{F}_{+}^{b}(X, Y)$ and the operator $\mathbb{Q}(\lambda) \in$ $\mathcal{F}_{+}(X, Y)$, then

$$
\sigma_{\text {eap }}(\mathcal{A}) \subseteq \sigma_{\text {eap }}\left(A_{1}\right) \cup \sigma_{\text {eap }}\left(\bar{M}_{\lambda}\right)
$$

If in the addition we suppose that the sets $\Phi_{\mathcal{A}}, \Phi_{A_{1}}$ and $\Phi_{\bar{M}_{\lambda}}$ are connected and the sets $\rho\left(\bar{M}_{\lambda}\right)$ and $\rho(\mathcal{A})$ are not empty, then:

$$
\sigma_{\text {eap }}(\mathcal{A})=\sigma_{\text {eap }}\left(A_{1}\right) \cup \sigma_{\text {eap }}\left(\bar{M}_{\lambda}\right) .
$$

(ii) If for some $\lambda \in \rho_{b}\left(A_{1}\right)$, the operator $C_{\lambda} \in \mathcal{F}_{-}^{b}(X, Y)$ and the operator $\mathbb{Q}(\lambda) \in$ $\mathcal{F}_{-}(X, Y)$, then

$$
\sigma_{e \delta}(\mathcal{A}) \subseteq \sigma_{e \delta}\left(A_{1}\right) \cup \sigma_{e \delta}\left(\bar{M}_{\lambda}\right) .
$$

If in the addition we suppose that the sets $\Phi_{\mathcal{A}}, \Phi_{A_{1}}$ and $\Phi_{\bar{M}_{\lambda}}$ are connected and the sets $\rho\left(\bar{M}_{\lambda}\right)$ and $\rho(\mathcal{A})$ are not empty, then

$$
\sigma_{e \delta}(\mathcal{A})=\sigma_{e \delta}\left(A_{1}\right) \cup \sigma_{e \delta}\left(\bar{M}_{\lambda}\right) .
$$

Proof Let $\mu \in \mathbb{C}$. Using the relation (3.7), we have

$$
\begin{aligned}
\mathcal{A}-\mu I & :=\mathbb{T}_{1}(\lambda) \mathbb{D}(\lambda) \mathbb{T}_{2}(\lambda)+\mathbb{N}(\lambda)+(\lambda-\mu) \\
& :=\mathbb{T}_{1}(\lambda) \mathbb{V}(\mu) \mathbb{T}_{2}(\lambda)+(\mu-\lambda) \mathbb{Q}(\lambda)-\mathbb{P}(\lambda)+\mathbb{N}(\lambda) .
\end{aligned}
$$

The matrices-operators $\mathbb{V}(\mu)$ and $\mathbb{P}(\lambda)$ are defined by

$$
\begin{gathered}
\mathbb{V}(\mu):=\left(\begin{array}{cc}
A_{1}-\mu I & 0 \\
0 & \bar{M}_{\lambda}-\mu I
\end{array}\right), \\
\mathbb{P}(\lambda):=\left(\begin{array}{cc}
{\left[A_{1}-(\lambda+1)\right] P_{\lambda}} & {\left[A_{1}-(\lambda+1)\right] P_{\lambda}\left[-\bar{K}_{\lambda} \bar{\Gamma}_{Y}^{0}+\overline{R_{b}\left(A_{1}, \lambda\right) B}\right]} \\
C_{\lambda}\left[A_{1}-(\lambda+1)\right] P_{\lambda} & C_{\lambda}\left[A_{1}-(\lambda+1)\right] P_{\lambda}\left[-\bar{K}_{\lambda} \bar{\Gamma}_{Y}^{0}+\overline{R_{b}\left(A_{1}, \lambda\right) B}\right]
\end{array}\right) .
\end{gathered}
$$

Since $\mathbb{T}_{1}(\lambda)$ and $\mathbb{T}_{2}(\lambda)$ are bounded and have bounded inverses, $\mathbb{N}(\lambda)$ and $\mathbb{P}(\lambda)$ are finite rank matrices operators and $\mathbb{Q}(\lambda) \in \mathcal{F}_{+}(X, Y)$, therefore, for the same reasons as the proof of Theorem 2.2, it follows from Eq. (3.9) that $(\mathcal{A}-\mu I)$ is an upper semi-Fredholm operator if only if $\mathbb{V}(\mu)$ has this property and $i(\mathcal{A}-\mu I)=i\left(A_{1}-\mu I\right)+i\left(\bar{M}_{\mu}-\mu I\right)$. This shows that

$$
\sigma_{\text {eap }}(\mathcal{A}) \subseteq \sigma_{\text {eap }}\left(A_{1}\right) \cup \sigma_{\text {eap }}\left(\bar{M}_{\lambda}\right) .
$$

Since $\Phi_{\mathcal{A}}, \Phi_{A_{1}}$ and $\Phi_{\bar{M}_{\lambda}}$ are connected and the sets $\rho\left(\bar{M}_{\lambda}\right)$ and $\rho(\mathcal{A})$ are not empty then, using Proposition 2.3 we get

$$
\sigma_{\text {eap }}(\mathcal{A})=\sigma_{\text {eg }}(\mathcal{A}), \sigma_{\text {eap }}\left(A_{1}\right)=\sigma_{\text {eg }}\left(A_{1}\right) \text { and } \sigma_{\text {eap }}\left(\bar{M}_{\lambda}\right)=\sigma_{\text {eg }}\left(\bar{M}_{\lambda}\right) .
$$

Now, the result follows from [19, Theorem 3.2(ii)] and the proof of $(i)$ is completed.

A same reasoning allows us to reach the result ( $i i)$. 


\section{Application to a two group of transport equations}

In this section, we will apply Theorem 3.3 to study the essential spectra of a class of linear operators on $L_{p}$-spaces, $1 \leq p<\infty$. Let

$$
X_{p}=L_{p}([-a, a] \times[-1,1], d x d \xi), \quad a>0 \text { and } p \in[1, \infty) .
$$

We consider the boundary spaces:

$$
X_{p}^{o}:=L_{p}[\{-a\} \times[-1,0],|\xi| d \xi] \times L_{p}[\{a\} \times[0,1],|\xi| d \xi]:=X_{1, p}^{o} \times X_{2, p}^{o}
$$

and

$$
X_{p}^{i}:=L_{p}[\{-a\} \times[0,1],|\xi| d \xi] \times L_{p}[\{a\} \times[-1,0],|\xi| d \xi]:=X_{1, p}^{i} \times X_{2, p}^{i},
$$

respectively, equipped with the norms

$$
\left\|\psi^{o}\right\|_{X_{p}^{o}}=\left(\left\|\psi_{1}^{o}\right\|_{X_{1, p}^{o}}^{p}+\left\|\psi_{2}^{o}\right\|_{X_{2, p}^{o}}^{p}\right)^{\frac{1}{p}}=\left[\int_{-1}^{0}|\psi(-a, \xi)|^{p}|\xi| d \xi+\int_{0}^{1}|\psi(a, \xi)|^{p}|\xi| d \xi\right]^{\frac{1}{p}}
$$

and

$$
\left\|\psi^{i}\right\|_{X_{p}^{i}}=\left(\left\|\psi_{1}^{i}\right\|_{X_{1, p}^{i}}^{p}+\left\|\psi_{2}^{i}\right\|_{X_{2, p}^{i}}^{p}\right)^{\frac{1}{p}}=\left[\int_{0}^{1}|\psi(-a, \xi)|^{p}|\xi| d \xi+\int_{-1}^{0}|\psi(a, \xi)|^{p}|\xi| d \xi\right]^{\frac{1}{p}} .
$$

Let $\mathcal{W}_{p}$ the space defined by:

$$
\mathcal{W}_{p}=\left\{\psi \in X_{p} \text { such that } \xi \frac{\partial \psi}{\partial x} \in X_{p}\right\}
$$

It is well-known that any function $\psi$ in $\mathcal{W}_{p}$ possesses traces on the spatial boundary $\{-a\} \times$ $(-1,0)$ and $\{a\} \times(0,1)$ which, respectively, belong to the spaces $X_{p}^{o}$ and $X_{p}^{i}$ (see [5]). They are denoted, respectively, by $\psi^{o}$ and $\psi^{i}$.

Now we will consider the matrix of operators

$$
\mathcal{A}=\mathcal{T}+\mathcal{K},
$$

where

$$
\mathcal{T} \psi=\left(\begin{array}{cc}
-\xi \frac{\partial \psi_{1}}{\partial x}-\sigma_{1}(\xi) \psi_{1} & 0 \\
0 & -\xi \frac{\partial \psi_{2}}{\partial x}-\sigma_{2}(\xi) \psi_{2}
\end{array}\right)=\left(\begin{array}{cc}
T_{1} & 0 \\
0 & T_{2}
\end{array}\right)\left(\begin{array}{l}
\psi_{1} \\
\psi_{2}
\end{array}\right)
$$

and

$$
\mathcal{K}=\left(\begin{array}{cc}
0 & K_{12} \\
K_{21} & K_{22}
\end{array}\right)
$$

with $K_{12}, K_{21}$ and $K_{22}$ are bounded linear operators defined on $X_{p}$ by

$$
\left\{\begin{aligned}
K_{i j}: X_{p} & \longrightarrow X_{p} \\
\psi & \longrightarrow \int_{-1}^{1} \kappa_{i j}\left(x, \xi, \xi^{\prime}\right) \psi\left(x, \xi^{\prime}\right) d \xi^{\prime}
\end{aligned}\right.
$$


and the kernels $\kappa_{12}(., .,),. \kappa_{21}(., .,$.$) and \kappa_{22}(., .,$.$) are assumed to be measurable. T_{1}$ is the operator defined by

$$
\left\{\begin{array}{l}
T_{1}: \mathcal{D}\left(T_{1}\right) \subseteq X_{p} \longrightarrow X_{p} \\
\psi \longrightarrow T_{1} \psi(x, \xi)=-\xi \frac{\partial \psi}{\partial x}(x, \xi)-\sigma_{1}(\xi) \psi(x, \xi) \\
\mathcal{D}\left(T_{1}\right)=\mathcal{W}_{p}
\end{array}\right.
$$

and $T_{2}$ is the streaming operator defined by

$$
\left\{\begin{array}{l}
T_{2}: \mathcal{D}\left(T_{2}\right) \subseteq X_{p} \longrightarrow X_{p} \\
\psi \longrightarrow T_{2} \psi(x, \xi)=-\xi \frac{\partial \psi}{\partial x}(x, \xi)-\sigma_{2}(\xi) \psi(x, \xi) \\
\mathcal{D}\left(T_{2}\right)=\left\{\psi \in \mathcal{W}_{p} \quad \text { such that } \psi^{i}=H\left(\psi^{o}\right)\right\},
\end{array}\right.
$$

where $\sigma(.) \in L^{\infty}(-1,1), \psi^{o}, \psi^{i}$ represent the outgoing and the incoming fluxes related by the boundary operator $H$ namely

$$
\left\{\begin{array}{l}
H: X_{1, p}^{o} \times X_{2, p}^{o} \longrightarrow X_{1, p}^{i} \times X_{2, p}^{i} \\
H\left(\begin{array}{l}
u_{1} \\
u_{2}
\end{array}\right)=\left(\begin{array}{ll}
H_{11} & H_{12} \\
H_{21} & H_{22}
\end{array}\right)\left(\begin{array}{l}
u_{1} \\
u_{2}
\end{array}\right)
\end{array}\right.
$$

with for $k, l \in\{1,2\}, H_{k l}: X_{l, p}^{0} \longrightarrow X_{k, p}^{i}, H_{k l} \in \mathcal{L}\left(X_{l, p}^{o}, X_{k, p}^{i}\right)$.

It is clear that the operator $\mathcal{T}$ is defined on $\mathcal{W}_{p} \times \mathcal{D}\left(T_{2}\right)$.

In the next, we will define the operator $\mathcal{A}$ on

$$
\mathcal{D}(\mathcal{A}):=\left\{\left(\begin{array}{l}
\psi_{1} \\
\psi_{2}
\end{array}\right) \text { such that } \psi_{1} \in \mathcal{W}_{p}, \psi_{2} \in \mathcal{D}\left(T_{H_{2}}\right) \text { and } \psi_{1}^{i}=\psi_{2}^{i}\right\} .
$$

Now, it is easy to check that $\Gamma_{X}$ and $\Gamma_{Y}$ are the following operators

$$
\left\{\begin{array}{c}
\Gamma_{X}: X_{p} \longrightarrow X_{p}^{i} \\
\psi \longrightarrow \psi^{i}
\end{array}\right.
$$

and

$$
\left\{\begin{array}{l}
\Gamma_{Y}: X_{p} \longrightarrow X_{p}^{i} \\
\psi_{2} \longrightarrow \psi^{i}=H \psi^{o}
\end{array}\right.
$$

Let $A_{1}$ the operator defined by

$$
\left\{\begin{array}{l}
A_{1}=T_{1} \\
\mathcal{D}\left(A_{1}\right)=\left\{\psi_{1} \in \mathcal{W}_{p} \text { such that } \psi_{1}^{i}=0\right\}
\end{array}\right.
$$

Let

$$
\lambda_{j}^{*}=\liminf _{|\xi| \rightarrow 0} \sigma_{j}(\xi), \quad j=1,2 .
$$


Remark 4.1 (i) For $\lambda$ such that $\operatorname{Re} \lambda>-\lambda_{1}^{*}$ (i.e., $\lambda \in \rho\left(A_{1}\right)$ ), it follows that the solution of

$$
\left(T_{1}-\lambda\right) \psi=0
$$

is formally given by

$$
\begin{aligned}
& \psi(x, \xi)=\psi(-a, \xi) e^{-\frac{\left(\lambda+\sigma_{1}(\xi)|a+x|\right.}{|\xi|}}, \quad \xi \in(0,1), \\
& \psi(x, \xi)=\psi(a, \xi) e^{-\frac{\left(\lambda+\sigma_{1}(\xi)|a-x|\right.}{|\xi|}}, \quad \xi \in(-1,0) .
\end{aligned}
$$

Thus, the operator $K_{\lambda}$ is defined on $X_{p}^{i}$ by

$$
\left\{\begin{array}{l}
K_{\lambda}: X_{p}^{i} \longrightarrow X_{p}, K_{\lambda} u:=\chi_{(0,1)}(\xi) K_{\lambda}^{+} u+\chi_{(-1,0)}(\xi) K_{\lambda}^{-} u \text { with } \\
\left(K_{\lambda}^{+} u\right)(x, \xi):=u(-a, \xi) e^{-\frac{\left(\lambda+\sigma_{1}(\xi)\right)|a+x|}{|\xi|}}, \quad \xi \in(0,1), \\
\left(K_{\lambda}^{-} u\right)(x, \xi):=u(a, \xi) e^{-\frac{\left(\lambda+\sigma_{1}(\xi)|a-x|\right.}{|\xi|}}, \quad \xi \in(-1,0),
\end{array}\right.
$$

where $\chi_{(-1,0)}($.$) and \chi_{(0,1)}($.$) denote, respectively, the characteristic functions of the in-$ tervals $(-1,0)$ and $(0,1)$. It is easy to see that the operator $K_{\lambda}$ is bounded and $\left\|K_{\lambda}\right\| \leq$ $\left(p \operatorname{Re} \lambda+\lambda_{1}^{*}\right)^{-\frac{1}{p}}$.

(ii) To verify that the operator $\mathbb{Q}(\lambda)$ defined in the third section is compact on $X_{p} \times X_{p}$, $1<p<\infty$ (resp. weakly compact on $X_{1} \times X_{1}$ ) we shall prove that the operators

$$
C_{\lambda}:=K_{21}\left(\lambda-A_{1}\right)^{-1}
$$

and

$$
-K_{\lambda} \Gamma_{Y}+\left(\lambda-A_{1}\right)^{-1} K_{12}
$$

are compact on $X_{p} \times X_{p}, 1<p<\infty$ (resp. weakly compact on $X_{1} \times X_{1}$ ).

Notice that the collision operators $K_{12}, K_{21}$ and $K_{22}$ defined in Eq. (4.2), act only on the velocity $\xi^{\prime}$, so $x$ may be seen, simply, as a parameter in $[-a, a]$. Then, we will consider each of these operators as a function

$$
K_{i j}(.): x \in[-a, a] \longrightarrow K(x) \in \mathcal{L}\left(L_{p}([-1,1] ; d \xi) .\right.
$$

Definition 4.1 A collision operator $K_{i j}$ in the form (4.2), is said to be regular if it satisfies the following conditions:

$$
\left\{\begin{array}{l}
\text { - the function } K_{i j}(.) \text { is measurable, } \\
\text { - there exists a compact subset } \mathcal{C} \subset \mathcal{L}\left(L_{p}([-1,1] ; d \xi)\right) \text { such that: } \\
\quad K_{i j}(x) \in \mathcal{C} \text { a.e. on }[-a, a], \\
-K_{i j}(x) \in \mathcal{K}\left(L_{p}([-1,1] ; d \xi)\right) \text { a.e. on }[-a, a],
\end{array}\right.
$$

where $\mathcal{K}\left(L_{p}([-1,1] ; d \xi)\right)$ is the set of compact operators on $L_{p}([-1,1], d \xi)$.

\section{Lemma 4.1 [21]}

(i) If $\frac{\kappa_{21}\left(x, \xi, \xi^{\prime}\right)}{\left|\xi^{\prime}\right|}$ defines a regular operator, then the operator $C_{\lambda}:=K_{21}\left(\lambda-A_{1}\right)^{-1}$ is weakly compact on $X_{1}$.

(ii) If $K_{21}$ is regular, then the operator $C_{\lambda}:=K_{21}\left(\lambda-A_{1}\right)^{-1}$ is compact on $X_{p}$ for $1<p<\infty$. 
(iii) If $K_{12}$ is regular, then the operator $\left(\lambda-A_{1}\right)^{-1} K_{12}$ is compact on $X_{p}$ for $1<p<\infty$ and weakly compact on $X_{1}$.

Remark 4.2 It follows from Theorem 3.1 in [23] that $\mathcal{W}\left(X_{1}\right)=\mathcal{S}\left(X_{1}\right)$.

If $1<p<\infty, X_{p}$ is reflexive and then $\mathcal{L}\left(X_{p}\right)=\mathcal{W}\left(X_{p}\right)$. On the other hand, it follows from [10, Theorem 5.2] that $\mathcal{K}\left(X_{p}\right) \subset_{\neq} \mathcal{S}\left(X_{p}\right) \subset_{\neq} \mathcal{W}\left(X_{p}\right)$ with $p \neq 2$. For $p=2$ we have $\mathcal{K}\left(X_{p}\right)=\mathcal{S}\left(X_{p}\right)=\mathcal{W}\left(X_{p}\right)$.

Theorem 4.1 If the operator $H \in \mathcal{S}\left(X_{p}\right)$ and the operators $K_{12}, K_{21}, K_{22}$ are regular and if in addition $\kappa_{21}\left(x, \xi, \xi^{\prime}\right)\left(\right.$ resp. $\left.\frac{\kappa_{21}\left(x, \xi, \xi^{\prime}\right)}{\left|\xi^{\prime}\right|}\right)$ defines a regular operator on $X_{p}$ for $1<p<\infty$ (resp. on $X_{1}$ ), then

$$
\sigma_{\text {eap }}(\mathcal{A})=\sigma_{e \delta}(\mathcal{A})=\left\{\lambda \in \mathbb{C} \text { such that } \operatorname{Re} \lambda \leq-\min \left(\lambda_{1}^{*}, \lambda_{2}^{*}\right)\right\} .
$$

Proof First, it is shown in [20] that

$$
\sigma_{\text {eap }}\left(A_{1}\right)=\sigma_{e \delta}\left(A_{1}\right)=\left\{\lambda \in \mathbb{C} \text { such that } \operatorname{Re} \lambda \leq-\lambda_{1}^{*}\right\} .
$$

Second, for $\lambda \in \rho\left(T_{2}\right)$ such that $r_{\sigma}\left(\left(\lambda-T_{2}\right)^{-1} K_{22}\right)<1$, then $\lambda \in \rho\left(T_{2}+K_{22}\right) \cap \rho\left(T_{2}\right)$ and we have,

$$
\left(\lambda-T_{2}-K_{22}\right)^{-1}-\left(\lambda-T_{2}\right)^{-1}=\sum_{n \geq 1}\left[\left(\lambda-T_{2}\right)^{-1} K_{22}\right]^{n}\left(\lambda-T_{2}\right)^{-1} .
$$

Since $K_{22}$ is regular, then it follows from [13, Lemma 3.1] that the operator $\left(\lambda-T_{2}-\right.$ $\left.K_{22}\right)^{-1}-\left(\lambda-T_{2}\right)^{-1}$ is compact on $X_{p}, 1<p<\infty$ and weakly compact on $X_{1}$. Then the use of Remark 3.3 in [20] leads to

$$
\sigma_{\text {eap }}\left(T_{2}+K_{22}\right)=\sigma_{\text {eap }}\left(T_{2}\right)=\left\{\lambda \in \mathbb{C} \text { such that } \operatorname{Re} \lambda \leq-\lambda_{2}^{*}\right\} .
$$

Let $\mu \in \rho(A)$. The operator $M_{\mu}$ is given by

$$
M_{\mu}=T_{2}+K_{22}+K_{21} K_{\mu} \Gamma_{Y}-K_{21}\left(\mu-A_{1}\right)^{-1} K_{12} .
$$

Since the operator $H$ is strictly singular on $X_{p}$ then $\Gamma_{Y}$ has also this property. This together with Lemma 4.1 make us conclude that $M_{\mu}-T_{2}-K_{22}$ is compact on $X_{p}, 1<p<\infty$ and weakly compact on $X_{1}$, then

$$
\sigma_{\text {eap }}\left(M_{\mu}\right)=\sigma_{\text {eap }}\left(T_{2}+K_{22}\right)=\left\{\lambda \in \mathbb{C} \text { such that } \operatorname{Re} \lambda \leq-\lambda_{2}^{*}\right\} .
$$

Applying Theorem 3.3, and using Eqs. (4.3) and (4.4) we get

$$
\sigma_{\text {eap }}(\mathcal{A})=\left\{\lambda \in \mathbb{C} \text { such that } \operatorname{Re} \lambda \leq-\min \left(\lambda_{1}^{*}, \lambda_{2}^{*}\right)\right\} .
$$

A same reasoning allows us to show that

$$
\sigma_{e \delta}(\mathcal{A})=\left\{\lambda \in \mathbb{C} \text { such that } \operatorname{Re} \lambda \leq-\min \left(\lambda_{1}^{*}, \lambda_{2}^{*}\right)\right\} .
$$

Remark 4.3 In this application, we have determined the essential approximate point spectrum and the essential defect spectrum of the matrix of $\mathcal{A}$ without knowing $\sigma_{e a p}(A)$ nor $\sigma_{e \delta}(A)$ because the domain of $A, \mathcal{D}(A)$, is maximal. But we know that the restriction of the operator $A$ on the intersection $\mathcal{D}(A) \cap \mathcal{N}\left(\Gamma_{X}\right)$ is a transport operator with vacuum boundary conditions. Hence, we can easily obtain the results and the application in [19] become a special case of our work. 
Open Access This article is distributed under the terms of the Creative Commons Attribution Noncommercial License which permits any noncommercial use, distribution, and reproduction in any medium, provided the original author(s) and source are credited.

\section{References}

1. Atkinson, F.V., Langer, H., Mennicken, R., Shkalikov, A.A.: The essential spectrum of some matrix operators. Math. Nachr. 167, 5-20 (1994)

2. Bátkai, A., Binding, P., Dijksma, A., Hryniv, R., Langer, H.: Spectral problems for operator matrices. Math. Nachr. 278, 1408-1429 (2005)

3. Ben Amar, A., Jeribi, A., Mnif, M.: Some results on Fredholm and semi-Fredholm perturbations and applications. preprint (2005)

4. Damak, M., Jeribi, A.: On the essential spectra of some matrix operators and application. Electron. J. Differ. Equ. 2007, 1-16 (2007)

5. Dautray, R., Lions, J.L.: Analyse mathématique et calcul numérique. Tome 9. Masson, Paris (1988)

6. Diestel, J.: Geomety of Banach Spaces-selected Topics. Lecture Notes in Mathematics, vol. 485. Springer, New York (1975)

7. Dunford, N., Pettis, B.J.: Linear operations on summable functions. Trans. Am. Math. Soc. 47, 323-392 (1940)

8. Dunford, N., Schwartz, J.T.: Linear Operators. Part I. General Theory. Wiley-Interscience, New York (1958)

9. Gohberg, I.C., Krein, I.M.: Fundamental theorems on deficiency numbers, root numbers and indices of linear operators. Am. Math. Soc. Transl. Ser. 213, 185-260 (1960)

10. Gohberg, I.C., Markus, A.S., Feldman, I.A.: Normally solvable operators and ideals associated with them. Am. Math. Soc. Transl. Ser. 261, 63-84 (1967)

11. Goldberg, S.: Unbounded Linear operators. McGraw-Hill, New York (1966)

12. Gustafson, K., Weidmann, J.: On the essential spectrum. J. Math. Anal. Appl. 25, 121-127 (1969)

13. Jeribi, A.: Quelques remarques sur les opérateurs de Fredholm et application à l'équation de transport. C. R. Acad. Sci. Paris, t. 325, Série I, pp. 43-48 (1997)

14. Jeribi, A.: A caracterisation of Schechter essential spectrum on Banach spaces and applications. J. Math. Anal. Appl. 271, 343-358 (2002)

15. Jeribi, A.: Some remarks on the Schechter essential spectrum and applications to a transport equation. J. Math. Anal. Appl. 275, 222-237 (2002)

16. Jeribi, A., Mnif, M.: Fredholm operators, essential spectra and application to transport equations. Acta Appl. Math. 89, 155-176 (2006)

17. Kato, T.: Perturbation theory for nullity, deficiency and other quantities of linear operators. J. Anal. Math. 6, 261-322 (1958)

18. Lutgen, J.: On essential spectra of operator-matrices and their Feshbach maps. J. Math. Anal. Appl. 289, 419-430 (2004)

19. Moalla, N., Damak, M., Jeribi, A.: Essential spectra of some matrix operators and some matrix operators and application to two-group transport operators with general boundary conditions. J. Math. Anal. Appl. 323, 1071-1090 (2006)

20. Moalla, N., Jeribi, A.: A characterization of some subsets of Schechter's essential spectrum and application to singular transport equation, preprint (2006)

21. Mokhatar-Kharroubi, M.: Time asymptotic behaviour and compactness in neutron transport theory. Eur. J Mech. B Fluid 11, 39-68 (1992)

22. Müller, V.: Spectral theory of linear operators and spectral systems in banach algebras. Operator theory advances and applications, vol. 139. Birrkhauser Verlag, Basel (2003)

23. Pelczynski, A.: Strictly singular and strictly cosingular operators. I. Strictly singular and strictly cosingular operators on $\mathcal{C}(\Omega)$-spaces. Bull. Acad. Polon. Sci. 31-36 (1965)

24. Rakočević, V.: On one subset of M. Schechter's Essential Spectrum. Math. Vesnik 5, 389-391 (1981)

25. Rakočević, V.: Approximate point spectrum and commuting compact perturbations. Glasgow Math. J. 28, 193-198 (1986)

26. Schechter, A.: On the essential spectrum of an arbitrary operator. J. Math. Anal. Appl. 13, 205-215 (1966)

27. Schechter, A.: Principles of Functionnal Analysis. Academic Press, New york (1971)

28. Schmoeger, C.: The spectral mapping theorem for the essential approximate point spectrum. Colloq. Math. 74(2), 167-176 (1997) 
29. Shannon, G.P.: Strictly singular and cosingular operators and topological vector spaces. Proc. R. Irish Acad. Sect. A 73, 303-308 (1973)

30. Shkalikov, A.A.: On the essential spectrum of some matrix operators. Math. Notes 58(6), 1359-1362 (1995)

31. Vladimirskii, Ju.I.: Strictly cosingular operators. Sov. Math. Dok1. 8, 739-740 (1967)

32. Weis, L.: Perturbation classes of semi-Fredholm operators. Math. Z. 178, 429-442 (1981)

33. Whitley, R.J.: Strictly singular operators and their conjugates. Trans. Am. Math. Soc. 18, 252-261 (1964)

34. Wolf, F.: On the invariance of the essential spectrum under a change of the boundary conditions of partial differential operators. Indag. Math. 21, 142-147 (1959) 